\title{
Acute Renal Failure and Its Risk Factors in Stevens-Johnson Syndrome and Toxic Epidermal Necrolysis
}

\author{
Chi-Chih Hung ${ }^{\mathrm{a}}$ Wan-Chun Liu ${ }^{\mathrm{a}}$ Mei-Chuan Kuo ${ }^{\mathrm{a}}$ Chih-Hung Lee ${ }^{\mathrm{b}}$ \\ Shang-Jyh Hwang a Hung-Chun Chen ${ }^{\text {a }}$ \\ ${ }^{a}$ Division of Nephrology, Department of Internal Medicine, and ${ }^{b}$ Department of Dermatology, Kaohsiung Medical \\ University, Kaohsiung, Taiwan, ROC
}

\section{Key Words}

Acute interstitial nephritis $\cdot$ Acute renal failure $\cdot$ Erythema multiforme $\cdot$ Hypersensitivity

\begin{abstract}
Background: Skin lesion is the most frequent manifestation of adverse drug reactions. Drug-induced cutaneous hypersensitivity and drug-induced acute interstitial nephritis might share a similar mechanism involving drug-specific $T$ cells. We thus investigated the renal outcome of StevensJohnson syndrome (SJS) and toxic epidermal necrolysis (TEN), the most severe drug-induced cutaneous hypersensitivity, and hypothesize that skin detachment in SJS/TEN might be associated with acute renal failure (ARF). Methods: 234 hospitalized patients were retrospectively classified into an SJS/TEN group (skin detachment) or an erythematous multiforme majus group (target-like exanthema alone). $\boldsymbol{R e}$ sults: Both drugs and chronic kidney disease (CKD) are associated with SJS/TEN. The SJS/TEN group was more likely to develop ARF than the erythematous multiforme majus group (18.8 vs. $4.3 \%, p<0.05)$ despite similar initial creatinine clearance. In the ARF patients, RIFLE-F class, dialysis and long-term dialysis were 25,15 and $5 \%$, respectively. The offending drugs in ARF were also associated with CKD. Hyponatremia and late hypokalemia were more frequently in the SJS/TEN group (15.6 vs. $2.9 \%, 7.3$ vs. $0.7 \%$, respectively, $p<$
\end{abstract}

0.05). Sepsis, allopurinol, antibiotics, NSAIDs, CKD and hypoalbuminemia (OR: 18.8, 9.8, 10.1, 9.0, 5.3 and 3.3, respectively, $p<0.05$ ) were the risk factors of developing ARF. Conclusion: ARF, the need for dialysis, and late hypokalemia could be the consequences of SJS/TEN. Skin detachment after certain medication might implicate the associated ARF, especially in CKD patients.

Copyright $\odot 2009$ S. Karger AG, Basel

\section{Introduction}

Skin is the most frequent target of adverse drug reactions, probably because of easy detection. Most of them are related to drug hypersensitivity [1]. Still, skin rash is not a frequent feature of drug-induced acute interstitial nephritis (AIN) [2]. The presence of skin lesions might be overlooked as only a skin reaction, rather than a systemic reaction. The relationship between skin and renal lesions in the context of drug hypersensitivity is little known. Hung et al. [3] showed that $50 \%$ of allopurinolinduced severe cutaneous adverse reactions were associated with worsening renal function. Then, if a skin lesion is observed in an adverse drug reaction, how much, how severe and what kind of renal lesions will there be? Because drug-induced AIN is fairly unpredictable, will the presentation of adverse skin reactions enable earlier no-

\section{KARGER}

Fax +41613061234 E-Mail karger@karger.ch www.karger.com
(C) 2009 S. Karger AG, Basel 0250-8095/09/0296-0633\$26.00/0

Accessible online at:

www.karger.com/ajn
Hung-Chun Chen, MD

Division of Nephrology, Department of Medicine

Kaohsiung Medical University

100 Tzyou First Road, Kaohsiung 807, Taiwan (ROC)

Tel. +886 7312 1101, Fax +886 7312 2810, E-Mail chenhc@kmu.edu.tw 
tification of renal injury? Furthermore, Spanou et al. [4] revealed that drug-specific $\mathrm{T}$ cells orchestrate a similar local inflammation and allergic reaction in both druginduced AIN and drug-induced cutaneous hypersensitivity. We would reasonably speculate that the severity of drug-induced AIN might be related to the severity of skin lesions.

Drug-induced cutaneous hypersensitivity presents with a variety of features. Stevens-Johnson syndrome (SJS) and toxic epidermal necrolysis (TEN) are the most severe and are characterized by a rapidly developing target-like exanthema accompanied by mucosal involvement and skin detachment [5]. These presentations differ from erythematous multiforme majus (EMM) which is related to infection and features only target-like exanthemas. SJS/TEN (denotes both SJS and TEN) and EMM are currently considered as cell-mediated reactions aimed at the destruction of keratinocytes expressing drug-related or herpes-related antigens, respectively [6]. Besides, more than $65 \%$ of SJS/TEN cases are induced by drugs including antibiotics, nonsteroid anti-inflammatory drugs (NSAIDs) and anticonvulsants [5]. Many of the offending drugs are well-known causes of AIN [2]. Case reports showed the co-existence of biopsy-proven AIN and SJS/ TEN [7]. Thus, we hypothesize that the characteristic skin detachment observed in SJS/TEN will predict renal injury in these patients, while the nonspecific target-like exanthema observed in EM will not. Also the renal manifestation in SJS/TEN is largely unknown. Drug, insensible water loss, sepsis and diarrhea might further complicate renal injury $[8,9]$. In view of the above, we carried out a retrospective study to investigate renal manifestations and risk factors of acute renal failure (ARF) in SJS/ TEN and to determine whether skin detachment in SJS/ TEN is associated with ARF.

\section{Methods}

\section{Patients}

We carried out a retrospective study of all adult cases admitted to Kaohsiung Medical University Hospital who were diagnosed as having EMM, SJS, overlap SJS/TEN, and TEN between January 1998 and June 2005 according to the classification of Bastuji-Garin et al. [10]. EMM patients were classified as the EMM group and all the other patients were classified as the SIS/TEN group [5, $8,10]$.

\section{Parameters Investigated}

The clinical information of cases and underlying diseases were recorded on a standardized form as listed in table 1. Chronic kidney disease (CKD; with estimated creatinine clearance, $\mathrm{Ccr}$,
Table 1. Clinical and demographic features

\begin{tabular}{lccl}
\hline Features & $\begin{array}{c}\text { SJS/TEN } \\
(\mathrm{n}=96)\end{array}$ & $\begin{array}{l}\text { EMM } \\
(\mathrm{n}=138)\end{array}$ & $\mathrm{p}$ \\
\hline Age, years & $53 \pm 2.0$ & $45 \pm 1.7$ & $\mathrm{NS}$ \\
$\begin{array}{l}\text { Male gender } \\
\text { Underlying diseases }\end{array} \quad 55.2 \%$ & $39.1 \%$ & $\mathrm{NS}$ \\
$\quad$ Diabetes mellitus & $15.6 \%$ & $10.1 \%$ & $\mathrm{NS}$ \\
$\quad$ Hypertension & $27.4 \%$ & $18.8 \%$ & $\mathrm{NS}$ \\
$\quad$ CKD & $38.2 \%$ & $22.3 \%$ & 0.015 \\
$\quad$ Cardiac disease & $7.3 \%$ & $0.7 \%$ & 0.009 \\
$\quad$ Chronic liver disease & $7.3 \%$ & $7.2 \%$ & $\mathrm{NS}$ \\
Temperature, ${ }^{\circ} \mathrm{C}$ & $37.3 \pm 0.1$ & $36.8 \pm 0.1$ & 0.001 \\
Patterns of EM & & & \\
$\quad$ Skin detachment, \% of BSA & $15.9 \pm 1.5$ & $0.6 \pm 0.2$ & $<0.001$ \\
$\quad$ Oral mucosa involvement & $88.5 \%$ & $18.1 \%$ & $<0.001$ \\
Drug associated (vs. infection) & $88.5 \%$ & $52.9 \%$ & $<0.001$ \\
\hline
\end{tabular}

$\mathrm{BSA}=$ Body surface area

below $60 \mathrm{ml} / \mathrm{min}$ ), cardiac disease (including arrhythmia, congestive heart failure and ischemic heart disease), and chronic liver disease (including chronic viral hepatitis and liver cirrhosis) were as defined.

ARF was classified according to the RIFLE GFR criteria [11]. Prerenal azotemia or intrinsic ARF was diagnosed based on the ratio of urea to creatinine (BUN:creatinine ratio) and response to hydration. Persistent hypokalemia was defined as serum $\mathrm{K}^{+}$level $\leq 3.0 \mathrm{mEq} / \mathrm{l}$ lasting for more than 2 weeks or requiring potassium supplement to maintain the serum $\mathrm{K}^{+}$level at $>3.5 \mathrm{mEq} / \mathrm{l}$.

\section{Statistical Analysis}

Data are presented either as actual numbers, percentage or mean \pm standard error of the mean. Differences between groups of cases were evaluated by the $\chi^{2}$ test, Fisher's exact test and Student's t test. A two-sided p value of 0.05 or less was considered as significant. Analyses and calculations were performed using SPSS statistical package, version 12.0 (SPSS Inc., Chicago, Ill., USA).

Multivariate logistic regression models were fitted with a forward stepwise algorithm. Parameter estimation was done by the likelihood ratio method. To test which demographic variables predict SJS/TEN lesion, we fitted into the logistic regression model all the recorded demographic variables. To test which laboratory and demographic variables predict ARF development, we chose the significant factors from univariate analysis for the logistic regression model.

\section{Results}

\section{Clinical and Demographic Features}

Among the 234 patients enrolled, 96 (41\%) and 138 (59\%) were classified as SJS/TEN and EMM, respectively (table 1). The SJS/TEN group differed significantly from 
Table 2. Distribution of associated drugs in subgroups

\begin{tabular}{|c|c|c|c|c|c|c|}
\hline Associated drugs & $\begin{array}{l}\text { SJS/TEN } \\
(\mathrm{n}=85)\end{array}$ & $\begin{array}{l}\text { EMM } \\
(\mathrm{n}=73)\end{array}$ & $\mathrm{p}$ & $\begin{array}{l}\text { CKD } \\
(\mathrm{n}=57)\end{array}$ & $\begin{array}{l}\text { Non-CKD } \\
(\mathrm{n}=101)\end{array}$ & $\mathrm{p}$ \\
\hline Carbamazepine $(\mathrm{n}=41)$ & 65.9 & 34.1 & $<0.001$ & 17.1 & 82.9 & 0.002 \\
\hline NSAIDs $(\mathrm{n}=34)$ & 61.8 & 38.2 & NS & 55.9 & 44.1 & 0.007 \\
\hline Antibiotics $(\mathrm{n}=20)$ & 50 & 50 & NS & 25 & 75 & NS \\
\hline Allopurinol $(\mathrm{n}=17)$ & 70.6 & 29.4 & NS & 82.4 & 17.6 & $<0.001$ \\
\hline Herbs $(n=11)$ & 45.5 & 54.5 & NS & 18.2 & 81.8 & NS \\
\hline Phenytoin $(\mathrm{n}=10)$ & 40 & 60 & NS & 50 & 50 & NS \\
\hline Other $(n=23)$ & 24 & 76 & 0.001 & 20 & 80 & NS \\
\hline
\end{tabular}

Figures represent percentages.

the EMM group in the clinical features of older age $(\mathrm{p}=$ $0.005)$, higher body temperature $(\mathrm{p}=0.001)$, and more associated drugs $(\mathrm{p}<0.001)$. These differences were compatible with previous observations [9]. In the SJS/TEN group, patients with CKD and cardiac disease also constituted a higher percentage than those in the EMM group (38.2 vs. $22.3 \%, \mathrm{p}=0.015 ; 7.3$ vs. $0.7 \%, \mathrm{p}=0.009$, respectively).

\section{Associated Drugs in Subgroups}

Drugs were the cause of SJS/TEN in $88.5 \%$ and of EMM in $52.9 \%$ of cases. Carbamazepine was the most common offending drug, followed by NSAIDs and antibiotics (table 2). These associated drugs are consistent with other SJS/TEN studies $[5,12]$. The allopurinol and NSAID subgroups had a higher percentage of CKD patients. In contrast, the carbamazepine subgroup had a higher percentage of non-CKD patients.

\section{Renal Manifestations and Laboratory Findings}

There was no difference in initial renal function between groups as shown by estimated Ccr (table 3). However, abnormal urinalysis, proteinuria and hematuria were higher in the SJS/TEN group (28.3 vs. $8.7 \%$, p < $0.001,32.6$ vs. $14.2 \%, \mathrm{p}=0.001$, respectively). The C-reactive protein level was also greater in the SJS/TEN group (56.1 vs. $27.0 \mathrm{mg} / \mathrm{dl}, \mathrm{p}<0.001$ ), but no difference in eosinophil count was seen.

The SJS/TEN group had a higher rate of ARF (20.8 vs. $4.3 \%, \mathrm{p}<0.001)$, but there was no difference in the need for dialysis (table 3). Each group had one case of mortality. Respiratory failure and septic shock were the causes of death for the SJS/TEN group and EMM group, respectively.

Renal Outcome in Stevens-Johnson Syndrome
Clinical Manifestations of ARF Patients

ARF patients had a higher frequency of CKD (85 vs. $23 \%, \mathrm{p}<0.001$ ), cardiac disease ( $12 \mathrm{vs.} 4 \%, \mathrm{p}=0.038$ ), offending drugs (96 vs. 64\%, $\mathrm{p}<0.001$ ) and sepsis (17 vs. $2 \%, \mathrm{p}<0.001$ ) than non-ARF patients (table 4 ). The interval between desquamation and admission was 4.55 days. $92 \%$ of ARF patients had a higher creatinine level than baseline on admission and $96 \%$ of them received corticosteroid treatment. The average peak creatinine level was about $3.44 \mathrm{mg} / \mathrm{dl}$, which took 18.9 days to return to baseline. The ARF patients also showed higher potassium, uric acid, eosinophil count and lower hemoglobin than the non-ARF patients. The types of ARF distributed equally as prerenal azotemia and intrinsic ARF. The factors predisposing to prerenal azotemia were noted as SJS/ TEN, sepsis, liver cirrhosis, colitis and bleeding. Three out of the $20 \mathrm{ARF}$ patients (15\%) required hemodialysis and one of them (5\%) entered long-term dialysis. In one mortality case, dialysis was indicated due to oliguria ARF, but dialysis was not undertaken because of terminal illness. Nearly all of them (95\%) had been prescribed offending drugs. Allopurinol (40\%) and NSAIDs (35\%) were the most commonly used.

\section{Electrolyte Imbalance}

Hyponatremia $(<130 \mathrm{mEq} / \mathrm{l})$ was found more frequently in the SJS/TEN group ( 15.6 vs. $2.9 \%, \mathrm{p}=0.001$ ) on admission, and all recovered in the follow-up (table 3 ). Hypokalemia was observed around 1-2 weeks after admission and more frequently in the SJS/TEN group (7.3 vs. $0.7 \%, p=0.009)$. Short-term polyuria was noted in 2 cases. Hyponatremia (18 cases) was associated with anticonvulsants, allopurinol, NSAIDs, herbs and ARF. Hypokalemia (8 cases) was associated with anticonvulsants,

Am J Nephrol 2009;29:633-638 
Table 3. Renal manifestations and the laboratory findings

\begin{tabular}{|c|c|c|c|}
\hline Variable & SJS/TEN $(\mathrm{n}=96)$ & $\operatorname{EMM}(\mathrm{n}=138)$ & $\mathrm{p}$ \\
\hline \multicolumn{4}{|l|}{ Renal function } \\
\hline Estimated CCr, $\mathrm{ml} / \mathrm{min}$ & $76.0 \pm 9.1$ & $81.5 \pm 3.3$ & NS \\
\hline BUN, mg/dl & $22.3 \pm 2.0$ & $16.3 \pm 1.1$ & 0.011 \\
\hline Creatinine, mg/dl & $1.57 \pm 0.21$ & $1.12 \pm 0.10$ & NS \\
\hline BUN:creatinine ratio & $16.0 \pm 6.8$ & $16.2 \pm 6.7$ & NS \\
\hline \multicolumn{4}{|l|}{ Urinalysis } \\
\hline Proteinuria & $28.3 \%$ & $8.7 \%$ & $<0.001$ \\
\hline Microscopic hematuria & $32.6 \%$ & $14.2 \%$ & 0.001 \\
\hline \multicolumn{4}{|l|}{ Electrolytes } \\
\hline $\mathrm{Na}, \mathrm{mEq} / \mathrm{l}$ & $135 \pm 0.6$ & $136 \pm 1.0$ & NS \\
\hline $\mathrm{K}, \mathrm{mEq} / \mathrm{l}$ & $4.29 \pm 0.07$ & $4.07 \pm 0.05$ & 0.01 \\
\hline Hyponatremia $(<130 \mathrm{mEq} / \mathrm{l})$ & $15.6 \%(15)$ & $2.9 \%(4)$ & 0.001 \\
\hline \multicolumn{4}{|l|}{ Other initial laboratory findings } \\
\hline $\mathrm{WBC}, / \mu \mathrm{l}$ & $8,671 \pm 382$ & $8,743 \pm 396$ & NS \\
\hline $\mathrm{Hgb}, \mathrm{g} / \mathrm{dl}$ & $13.1 \pm 0.2$ & $12.9 \pm 0.2$ & NS \\
\hline Eosinophil, \% & $3.4 \pm 0.6$ & $3.4 \pm 0.4$ & NS \\
\hline C-reactive protein, $\mathrm{mg} / \mathrm{dl}$ & $56.1 \pm 6.4$ & $27.0 \pm 3.3$ & $<0.001$ \\
\hline Albumin, g/dl & $3.62 \pm 0.06$ & $3.87 \pm 0.04$ & 0.002 \\
\hline GPT, IU/1 & $66.4 \pm 10.0$ & $54.4 \pm 8.2$ & NS \\
\hline \multicolumn{4}{|l|}{ Renal consequences } \\
\hline Acute renal failure & $20.8 \%(20)$ & $4.3 \%(6)$ & 0.001 \\
\hline Late hypokalemia $^{1}$ & $7.3 \%(7)$ & $0.7 \%(1)$ & 0.009 \\
\hline Polyuria & $2.0 \%(2)$ & $0 \%(0)$ & NS \\
\hline Need of Dialysis & $3.1 \%(3)$ & $1.4 \%(2)$ & NS \\
\hline Corticosteroid treatment & $99.0 \%$ & $94.2 \%$ & NS \\
\hline Mortality & $1 \%(1)$ & $0.7 \%(1)$ & NS \\
\hline
\end{tabular}

To convert BUN in $\mathrm{mg} / \mathrm{dl}$ to $\mathrm{mM}$, multiply by 0.357 ; creatinine in $\mathrm{mg} / \mathrm{dl}$ to $\mathrm{mM}$, multiply by 0.088 .

${ }^{1}$ Newly developed hypokalemia during hospitalization as defined in 'Methods'.
NSAIDs, and allopurinol. These hypokalemic cases will be discussed in greater detail in another article. Three cases had transtubular potassium gradient exceeding 4 and 3 cases had ARF. No diuretics use, diarrhea, poor nutrition or active skin detachment were observed in the same period. Hypokalemia persisted from 2 weeks to 5 years and potassium supplement was needed.

\section{Risk Factors of Renal and Skin Outcome}

In multivariate analysis, independent risk factors were listed (table 5). Drugs (OR: 7.41), CKD (OR: 2.19) and male gender (OR: 2.19) were the risk factors of SJS/TEN in all patients. Sepsis (OR: 18.82), allopurinol (OR: 9.79), antibiotics (OR: 10.09), NSAIDs (OR: 9.03), CKD (OR: 5.3) and hypoalbuminemia (albumin OR: 0.3) were the risk factors of developing $\mathrm{ARF}$ in all patients.

\section{Discussion}

We demonstrated for the first time the high frequency of ARF (20.8\%), the need for dialysis (3.1\%), and hypokalemia (7.3\%) in SJS/TEN patients. The incidence of ARF or hypokalemia is significantly higher in SJS/TEN patients than in EMM patients. Most of the patients were hospitalized for 5 days after the onset of desquamation and presented elevated serum creatinine at the time of admission. The use of allopurinol, NSAIDs, and antibiotics are associated with higher risk of ARF. Thus, the presence of characteristic skin detachment in adverse drug reaction should be regarded as a sign of the renal injury. Furthermore, we found that up to $25 \%$ of the ARF patients are classified as RIFLE - F class. 15 and $5 \%$ of the ARF patients required temporary dialysis and long-term dialysis, respectively. Hypokalemia, partly due to potassium-losing nephropathy, may last for years. Thus, SJS/ TEN could result in profound renal injury and should be 
Table 4. Characteristics of ARF in SJS/TEN patients

\begin{tabular}{lc}
\hline Features & \\
\hline CKD stage 3-5 & \\
Peak creatinine, mg/dl & $85 \%(17)$ \\
RIFLE classification & $3.44 \pm 0.8$ \\
$\quad$ R+I class & $75 \%(15)$ \\
$\quad$ F class & $25 \%(5)$ \\
Duration of ARF, days & $18.9 \pm 5.6$ \\
Desquamation to admission interval, days & $4.55 \pm 0.8$ \\
Type of ARF & \\
$\quad$ Prerenal azotemia & $40 \%(8)$ \\
$\quad$ Intrinsic ARF & $45 \%(9)$ \\
$\quad$ Multifactorial ARF & $15 \%(3)$ \\
Need for dialysis & $15 \%(3)$ \\
$\quad$ Long-term dialysis & $5 \%(1)$ \\
Duration of temporary HD, days & 16 \\
Offending drugs & $95 \%(19)$ \\
$\quad$ Allopurinol & $40 \%(8)$ \\
NSAIDs & $35 \%(7)$ \\
Antibiotics & $10 \%(2)$ \\
Anticonvulsants & $10 \%(2)$ \\
Mortality & $5 \%(1)$ \\
\hline
\end{tabular}

Table 5. Risk factors of SJS/TEN and ARF in all patients

\begin{tabular}{llrlr}
\hline Outcome & Risk factors & OR & 95\% CI & $\mathrm{p}$ \\
\hline SJS/TEN $^{1}$ & Drug (vs. infection) & 7.41 & $2.89-19.0$ & $<0.001$ \\
& CKD & 2.19 & $1.08-4.45$ & 0.030 \\
& Male sex & 1.97 & $1.01-3.86$ & 0.048 \\
\hline ARF $^{2}$ & Sepsis & 18.82 & $2.29-155.0$ & 0.006 \\
& Allopurinol & 9.79 & $1.40-68.2$ & 0.021 \\
& Antibiotics & 10.09 & $1.27-80.14$ & 0.029 \\
& NSAIDs & 9.03 & $1.40-58.25$ & 0.021 \\
& CKD & 5.30 & $1.33-21.12$ & 0.018 \\
& Albumin & 0.30 & $0.18-0.48$ & $<0.001$ \\
\hline
\end{tabular}

\footnotetext{
${ }^{1}$ Hosmer-Lemeshow goodness of fit test: $\chi=8.66$, d.f. $=5$, $\mathrm{p}=0.12$.

${ }^{2}$ Hosmer-Lemeshow goodness of fit test: $\chi=8.81$, d.f. $=8$, $\mathrm{p}=0.36$.
}

considered as a cause of mixed intrinsic and extrinsic ARF.

We then demonstrated that sepsis, associated drugs, CKD and hypoalbuminemia are the independent risk factors of ARF. To our surprise, skin detachment or mucosal involvement are not independently associated with ARF in total group or subgroup analysis. These results might be related to the high prevalence of CKD in SJS/ TEN patients (38\%) and high incidence of ARF among these CKD patients (49\%). We found for the first time the association between CKD and the development of STS/ TEN and the association between CKD and allopurinol or NSAIDs in our groups. We suspect the dominant effect of CKD may make STS/TEN patients prone to develop ARF. These might suggest either the high exposure of allopurinol and NSAIDs in CKD patients or high incidence of SJS/TEN in CKD patients. CKD could increase the risk of adverse drug reactions by changing drug metabolism via altering renal and nonrenal clearance, protein binding and volume of distribution [13]. It is not clear whether CKD patients would have a higher rate of drug hypersensitivity. Further study is needed to clarify the relationship between drug-induced AIN and drug-induced STS/TEN.

Prerenal azotemia indicated by elevated serum urea and tachycardia was identified as an independent risk factor for death in TEN [3]. ARF in SJS was considered a result of increased insensible water loss from skin and fluid loss from the gastrointestinal tract. However, in our groups, the consequences of skin and mucosal lesions, sepsis and hypoalbumin are independently associated with ARF. In our series, 8 out of 8 sepsis cases had ARF and 2 required dialysis. Liano et al. [14] reported that sepsis caused acute tubular necrosis in $27-35 \%$ of hospitalized patients. In addition, hypoalbuminemia, an indicator of malnutrition and possible decreased effective volume, is a strong predictor of ARF in our series and also in other studies with ICU patients [15]. As a consequence, appropriate effective volume should be kept.

Allopurinol, antibiotics and NSAIDs increased the risk for ARF in our multivariate analysis. We found that $50 \%$ of allopurinol-induced SJS/TEN patients suffered from ARF, which is in agreement with a previous observation (between 33 and 85\%) [3]. Allopurinol is well known for its hypersensitivity syndrome consisting of an erythematous skin rash, fever, hepatitis, eosinophilia, and worsening renal function. Although carbamazepine is the leading cause of SJS/TEN in our series, there is only one ARF case in this group. Carbamazepine and allopurinol are the major causes of SJS/TEN in Asian patients, accounting for $25-33$ and $25-37 \%$ cases, respectively, which might be related to specific HLA typing in the Chinese population [16-18]. Despite their similarity in frequency and genetic predisposition, they show differences in ARF susceptibility. We suspect this may be related to the high prevalence of CKD in allopurinol but not in the carbamazepine subgroup, as shown in table 2. Allopuri- 
nol and oxipurinol are metabolized via the kidneys, whereas the metabolism of carbamazepine depends on the detoxification capacity of hepatocyte [19].

We also demonstrated that initial hyponatremia (15.6\%) and late hypokalemia (7.3\%) could be manifestations of SJS/TEN patients. Anticonvulsants, NSAIDs and ARF are well known for the induction of syndrome of inappropriate antidiuretic hormone secretion [20]. Hyponatremia resolved quickly without any problem. In contrast, hypokalemia was not detected initially and developed within 1-2 weeks with or without the occurrence of ARF. NSAIDs, anticonvulsants (phenytoin and carbamazepine), and allopurinol, which do not have hypokalemic effects by themselves, might indirectly lead to hypokalemia in these cases. Here we consider that drug hypersensitivity-related potassium-losing nephropathy might be one of the causes.

In conclusion, we have demonstrated a high frequency of ARF, the need for dialysis, and hypokalemia in the hospitalized SJS/TEN patients. The incidence of ARF or hypokalemia is significantly higher in SJS/TEN patients than in EMM patients. Sepsis, associated drugs (allopurinol, NSAIDs and antibiotics), CKD and hypoalbuminemia are the independent risk factors of ARF in SJS/TEN patients. Thus, skin detachment presented after certain medication may implicate the associated ARF, especially in CKD patients.

\section{References}

1 Roujeau JC: Clinical heterogeneity of drug hypersensitivity. Toxicology 2005;209:123129.

2 Clarkson MR, Giblin L, O'Connell FP, O'Kelly P, Walshe JJ, Conlon P, O’Meara Y, Dormon A, Campbell E, Donohoe J: Acute interstitial nephritis: clinical features and response to corticosteroid therapy. Nephrol Dial Transplant 2004;19:2778-2783.

$\checkmark 3$ Hung SI, Chung WH, Liou LB, Chu CC, Lin M, Huang HP, Lin YL, Lan JL, Yang LC, Hong HS, Chen MJ, Lai PC, Wu MS, Chu CY, Wang KH, Chen CH, Fann CS, Wu JY, Chen YT: HLA-B*5801 allele as a genetic marker for severe cutaneous adverse reactions caused by allopurinol. Proc Natl Acad Sci USA 2005;102:4134-4139.

-4 Spanou Z, Keller M, Britschgi M, Yawalkar N, Fehr T, Neuweiler J, Gugger M, Mohaupt $\mathrm{M}$, Pichler WJ: Involvement of drug-specific $\mathrm{T}$ cells in acute drug-induced interstitial nephritis. J Am Soc Nephrol 2006;17:29192927.

5 Auquier-Dunant A, Mockenhaupt M, Naldi L, Correia O, Schroder W, Roujeau JC: Correlations between clinical patterns and causes of erythema multiforme majus, Stevens-Johnson syndrome, and toxic epidermal necrolysis: results of an international prospective study. Arch Dermatol 2002;138: 1019-1024.
6 Caproni M, Torchia D, Schincaglia E, Volpi W, Frezzolini A, Schena D, Marzano A, Quaglino P, De SC, Parodi A, Barletta E, Fabbri $\mathrm{P}$ : The CD40/CD40 ligand system is expressed in the cutaneous lesions of erythema multiforme and Stevens-Johnson syndrome/ toxic epidermal necrolysis spectrum. Br J Dermatol 2006;154:319-324.

7 Hsu SI: Biopsy-proved acute tubulointerstitial nephritis and toxic epidermal necrolysis associated with vancomycin. Pharmacotherapy 2001;21:1233-1239.

-8 Bastuji-Garin S, Fouchard N, Bertocchi M, Roujeau JC, Revuz J, Wolkenstein P: SCORTEN: a severity-of-illness score for toxic epidermal necrolysis. J Invest Dermatol 2000;115:149-153.

$\checkmark 9$ Wolkenstein P, Revuz J: Drug-induced severe skin reactions. Incidence, management and prevention. Drug Saf 1995;13:56-68.

10 Bastuji-Garin S, Rzany B, Stern RS, Shear NH, Naldi L, Roujeau JC: Clinical classification of cases of toxic epidermal necrolysis, Stevens-Johnson syndrome, and erythema multiforme. Arch Dermatol 1993;129:9296.

11 Bellomo R, Ronco C, Kellum JA, Mehta RL, Palevsky P: Acute renal failure - definition, outcome measures, animal models, fluid therapy and information technology needs: the Second International Consensus Conference of the Acute Dialysis Quality Initiative (ADQI) Group. Crit Care 2004;8:R204R212.

12 Lin MS, Dai YS, Pwu RF, Chen YH, Chang NC: Risk estimates for drugs suspected of being associated with Stevens-Johnson syndrome and toxic epidermal necrolysis: a case-control study. Intern Med J 2005;35: $188-190$.
13 Dreisbach AW, Lertora JJ: The effect of chronic renal failure on hepatic drug metabolism and drug disposition. Semin Dial 2003; 16:45-50.

14 Liano F, Junco E, Pascual J, Madero R, Verde $\mathrm{E}$ : The spectrum of acute renal failure in the intensive care unit compared with that seen in other settings. The Madrid Acute Renal Failure Study Group. Kidney Int Suppl 1998; 66:S16-S24.

15 Yegenaga I, Hoste E, Van BW, Vanholder R, Benoit D, Kantarci G, Dhondt A, Colardyn F, Lameire N: Clinical characteristics of patients developing ARF due to sepsis/systemic inflammatory response syndrome: results of a prospective study. Am J Kidney Dis 2004; 43:817-824

16 Yeung CK, Ma SY, Hon C, Peiris M, Chan $\mathrm{HH}$ : Aetiology in sixteen cases of toxic epidermal necrolysis and Stevens-Johnson syndrome admitted within eight months in a teaching hospital. Acta Derm Venereol 2003; 83:179-182.

17 Chan HL: Toxic epidermal necrolysis in Singapore, 1989 through 1993: incidence and antecedent drug exposure. Arch Dermatol 1995;131:1212-1213.

18 Chung WH, Hung SI, Hong HS, Hsih MS, Yang LC, Ho HC, Wu JY, Chen YT: Medical genetics: a marker for Stevens-Johnson syndrome. Nature 2004;428:486.

19 Krauss G: Current understanding of delayed anticonvulsant hypersensitivity reactions. Epilepsy Curr 2006;6:33-37.

20 Adrogue HJ, Madias NE: Hyponatremia. N Engl J Med 2000;342:1581-1589. 